\title{
REMOÇÃO DE CHUMBO(II) EM SISTEMAS DESCONTÍNUOS POR CARVÕES ATIVADOS COM ÁCIDO FOSFÓRICO E COM VAPOR
}

\author{
Cristiane Imenes de Campos Bueno e Wagner Alves Carvalho*
}

Faculdade de Química, Centro de Ciências Exatas, Ambientais e de Tecnologias, Pontifícia Universidade Católica de Campinas, Rod. D. Pedro I, km 136, 13086-900 Campinas - SP, Brasil

Recebido em 5/12/06; aceito em 18/5/07; publicado na web em 25/10/07

\begin{abstract}
LEAD(II) REMOVAL IN DISCONTINOUS SYSTEMS BY CARBON ACTIVATED BY PHOSPHORIC ACID AND VAPOR. Adsorption of heavy metal cations by activated carbon is dependent on the capacity of the material in promoting adsorption and the time needed to reach equilibrium. Carbon samples were previously activated either by phosphoric acid treatment at $400{ }^{\circ} \mathrm{C}$ or by steam at $800{ }^{\circ} \mathrm{C}$. The results of $\mathrm{Pb}$ (II) adsorption by these activated carbons have shown that equilibrium was typically reached within the first $5 \mathrm{~min}$ of contact between carbon and metal solution, with a maximum adsorption capacity higher than $69 \mathrm{mg} \mathrm{g}^{-1}$ for the vaporactivated sample. Temperature influences the sorption capacity, which corresponds to an endothermic process. Lead(II) retention is more pronounced at high temperature and low $\mathrm{pH}$.
\end{abstract}

Keywords: adsorption; activated carbon; lead(II).

\section{INTRODUÇÃO}

No meio industrial, uma atenção especial vem sendo dada aos impactos gerados pela utilização de metais potencialmente tóxicos. Essas espécies, quando lançadas no meio ambiente sem tratamento adequado, podem contaminar o solo, o subsolo e os lençóis freáticos. Contudo, a poluição industrial afeta diretamente o homem, uma vez que estamos sujeitos a consumir água e alimentos contaminados, o que pode causar sérios problemas à saúde.

Entre os poluentes mais prejudiciais ao ecossistema estão os metais pesados ou potencialmente tóxicos $(\mathrm{Pb}, \mathrm{Ni}, \mathrm{Cr}, \mathrm{Cd}$, $\mathrm{Hg}, \mathrm{Cu}$ e $\mathrm{Zn}$ são elementos considerados metais pesados, pois apresentam elevada densidade e geralmente estão associados a processos de contaminação). Tais elementos existem naturalmente no ambiente e muitos são necessários em concentrações mínimas na manutenção da saúde dos seres vivos (são denominados oligoelementos ou micronutrientes). No entanto, quando ocorre o aumento destas concentrações, normalmente acima de 10 vezes, efeitos deletérios começam a surgir. Metais potencialmente tóxicos em excesso podem causar muitas doenças e sérios problemas fisiológicos, já que são cumulativos no corpo humano ${ }^{1}$.

Outro aspecto importante a respeito da presença de metais em ambientes aquáticos diz respeito à forma com que a espécie metálica se encontra em solução. Os elementos metálicos diferenciamse pela quantidade na qual estão presentes, mas também em função das interações que promovem com outras espécies dissolvidas. Isto significa que um metal pode estar presente em um corpo d'água em várias formas físico-químicas diferentes ${ }^{2}$. A forma físico-química como um metal se apresenta é chamada de especiação química $^{3}$. A importância da compreensão da especiação química de um elemento metálico deve-se ao fato dela influenciar as propriedades, a disponibilidade biológica e, conseqüentemente, a toxicidade do metal tanto em águas naturais quanto em águas residuárias.

*e-mail: wagneracr@puc-campinas.edu.br
Dentre os metais potencialmente tóxicos mais preocupantes por sua toxicidade e larga utilização tem-se o chumbo. A contaminação da água pelo chumbo tem sido objeto de várias pesquisas. Água potável com baixo $\mathrm{pH}$ e baixas concentrações de sais dissolvidos pode carregar quantidades de chumbo vindas de soldas, encanamentos e ferragens, cisternas e reservatórios. O limite máximo de descarte de chumbo em corpos receptores permitido pela legislação é de $0,5 \mathrm{mg} \mathrm{L}^{-1}$ (Conselho Nacional de Meio Ambiente - CONAMA, Resolução no 357/2005).

O tratamento clássico de efluentes contendo íons de metais potencialmente tóxicos envolve processos físico-químicos como a precipitação química, podendo ser feita, por exemplo, pela adição de uma base (geralmente hidróxido de cálcio) ao efluente, de modo que haja a formação de espécies metálicas insolúveis sob a forma de hidróxidos e/ou óxidos. Processos subseqüentes de sedimentação e filtração são então realizados para que, posteriormente, a água tratada possa ser recuperada. Contudo, estas técnicas tradicionais são inadequadas para a descontaminação de grandes volumes de efluentes contendo íons de metais potencialmente tóxicos em baixas concentrações, devido a baixa eficiência operacional e aos elevados custos de extração resultante deste processo ${ }^{4}$.

Custo é um importante parâmetro na procura e comparação entre materiais adsorventes. De maneira geral, materiais de baixo custo estão relacionados àqueles que são abundantes na natureza e requerem pouco ou nenhum processamento prévio para sua utilização ${ }^{5}$. Também podem ser incluídos os subprodutos e resíduos oriundos das atividades agrícola e industrial ${ }^{6}$. Diversos trabalhos têm investigado o uso destes materiais na adsorção de metais potencialmente tóxicos. No Brasil, a abundância de alguns destes adsorventes incentivou estudos sobre a sua aplicação, dentre os quais destacamos quitosana ${ }^{7}$, zeólitas $^{8,9}, \operatorname{argilas}^{10}$, biomassa $^{11}$ e turfa $^{12}$. Em um país com elevado potencial de produção de carvão, o seu uso como adsorvente, principalmente na forma ativada, também deve ser considerado.

O uso do carvão ativado na remoção de compostos inorgânicos divide-se basicamente em três áreas: indústria metalúrgica, química analítica e tratamento de água e efluentes. Também é possível 
identificar a sua aplicação na indústria alimentícia e de bebidas ${ }^{13}$. A aplicação do carvão ativado no tratamento de efluentes está relacionada principalmente à redução de material orgânico, em especial de espécies que alteram a cor de efluentes. Por outro lado, muito pouco desses estudos tem sido direcionado ao uso do carvão ativado para a remoção de compostos inorgânicos em água e efluentes aquosos de indústrias ${ }^{14}$.

Neste trabalho, avaliou-se a capacidade de retenção de chumbo(II) presente em meio aquoso por dois carvões ativados brasileiros, produtos comerciais fornecidos pela Brascarbo Agroindustrial Ltda. (Paraná, Brasil) e identificados como Clarimex 061 CAE (carvão ativado com ácido fosfórico a $400{ }^{\circ} \mathrm{C}$ ) e CarboActiv V-plus (carvão ativado com vapor d'água a $800^{\circ} \mathrm{C}$ ).

\section{PARTE EXPERIMENTAL}

\section{Titulação de Boehm}

As concentrações relativas dos diferentes grupos funcionais de superfície foram determinadas pelo método de Boehm ${ }^{15}$, no qual amostras de carvão são mantidas em contato com soluções aquosas de $\mathrm{NaHCO}_{3}, \mathrm{NaOH}, \mathrm{CH}_{3} \mathrm{CH}_{2} \mathrm{ONa}$ ou $\mathrm{Na}_{2} \mathrm{CO}_{3}$ por $72 \mathrm{~h}$ em temperatura ambiente. Alíquotas da fase líquida de cada sistema são, então, tituladas com $\mathrm{HCl} 0,1 \mathrm{~mol} \mathrm{~L}^{-1}$.

\section{Titulação de pH}

Na titulação de pH foi utilizado um método adaptado de Strelko e Malik ${ }^{16}$. Amostras de carvão ativado suspensas em $20 \mathrm{~mL}$ de $\mathrm{NaCl}$ $0,1 \mathrm{~mol} \mathrm{~L}^{-1}$ foram postas em contato com soluções aquosas contendo quantidades variáveis de $\mathrm{NaOH}$ ou de $\mathrm{HCl} 0,1 \mathrm{~mol} \mathrm{~L}^{-1}$. Após $72 \mathrm{~h}$, o $\mathrm{pH}$ resultante foi medido. A quantidade de prótons adsorvida (Q) foi calculada usando-se a Equação 1.

$Q=\frac{1}{m}\left(V_{0}+V_{t}\right)\left([H]_{i}-[O H]_{i}-[H]_{e}+[O H]_{e}\right)$

onde $\mathrm{V}_{0}$ e $\mathrm{V}_{\mathrm{t}}$ são, respectivamente, o volume inicial de amostra e o volume do titulante adicionado, $m$ é a massa do adsorvente. Os subscritos $i$ e $e$ referem-se às concentrações de prótons e hidroxilas iniciais e no equilíbrio, respectivamente. Um pHmetro digital Analyser modelo 300M foi usado nas medidas de $\mathrm{pH}$.

\section{Adsorção de nitrogênio}

As medidas de adsorção de nitrogênio foram realizadas em um aparelho Micromeritics ASAP 2010 a uma temperatura de $-196{ }^{\circ} \mathrm{C}$, sob pressões na faixa de 10 a $925 \mathrm{mmHg}$. A área específica dos catalisadores foi determinada pelo método de Brunauer, Emmett e Teller (BET). O método $t$-plot foi utilizado para avaliar o volume microporoso, enquanto a mesoporosidade foi calculada de acordo com o método de Barrett, Joyner e Halenda (BJH).

\section{Curva analítica}

Soluções de $\mathrm{Pb}(\mathrm{II})$ com concentrações na faixa de 1,0 a 20,0 mg $\mathrm{L}^{-1}$ foram preparadas a partir de uma solução padrão de $1000 \mathrm{mg} \mathrm{L}^{-1}$ do metal (Titrisol ${ }^{\circledR}$, Merck) e utilizadas para a construção da curva analítica. A quantificação do metal em solução aquosa foi feita por espectrofotometria de absorção atômica (EAA), em um espectrofotômetro Perkin Elmer modelo Analyst 300. Na faixa de concentração estudada o comportamento observado é linear, sendo o coeficiente de correlação igual a 0,9990 .

\section{Preparo das soluções de $\mathbf{P b}(\mathrm{II})$}

Uma solução com concentração de $\mathrm{Pb}(\mathrm{II})$ equivalente a 1000 $\mathrm{mg} \mathrm{L}^{-1}$ foi preparada utilizando-se nitrato de chumbo(II) p.a. (99\%, Aldrich). A partir da diluição desta, foram preparadas soluções com concentração variando de $10 \mathrm{a} 1000 \mathrm{mg} \mathrm{L}^{-1}$. A concentração das soluções foi verificada por EAA.

\section{Cinética de adsorção}

Amostras contendo $0,8 \mathrm{~g}$ de carvão ativado em pó CarboActiv V-plus foram postas em contato com 120,0 mL da solução de $\mathrm{Pb}(\mathrm{II})$ de concentração $594 \mathrm{mg} \mathrm{L}^{-1}$.

O sistema foi mantido sob agitação magnética constante durante $20 \mathrm{~min}$, a $25^{\circ} \mathrm{C}$. Alíquotas de $5 \mathrm{~mL}$ foram recolhidas em intervalos de tempo na faixa de 1 a 20 min de agitação. Em seguida, as amostras coletadas foram filtradas em filtro RC15 com $0,2 \mu \mathrm{m}$ de diâmetro de poros, acoplado a uma seringa de vidro. $\mathrm{O}$ teor de $\mathrm{Pb}$ (II) nas soluções foi avaliado por EAA. O procedimento foi repetido com 120,0 mL da solução de $50 \mathrm{mg} \mathrm{L}^{-1}$ e 2,0 g do carvão ativado Clarimex 061 CAE.

\section{Isotermas de adsorção}

Amostras contendo 0,04 g de carvão ativado em pó CarboActiv V-plus foram postas em contato com $6,0 \mathrm{~mL}$ das soluções de $\mathrm{Pb}$ (II) com concentrações variando de 10 a $1000 \mathrm{mg} \mathrm{L}^{-1}$. Os sistemas foram mantidos sob agitação magnética contínua por $10 \mathrm{~min}$, a $25^{\circ} \mathrm{C}$. Em seguida, alíquotas foram filtradas e quantificadas por EAA.

O procedimento foi repetido com $0,1 \mathrm{~g}$ de carvão ativado Clarimex $061 \mathrm{CAE}$ em 6,0 mL das soluções de $\mathrm{Pb}(\mathrm{II})$.

\section{Influência da temperatura}

A influência da temperatura na cinética de adsorção foi avaliada em quatro diferentes temperaturas $\left(5,15,25\right.$ e $\left.50{ }^{\circ} \mathrm{C}\right)$, utilizando $60 \mathrm{~mL}$ de solução com concentração $50 \mathrm{mg} \mathrm{L}^{-1} \mathrm{de} \mathrm{Pb}(\mathrm{II})$ em $1,0 \mathrm{~g}$ de carvão ativado Clarimex 061 CAE ou $60 \mathrm{~mL}$ de solução $594 \mathrm{mg} \mathrm{L}^{-1}$ de $\mathrm{Pb}(\mathrm{II})$ em 0,4 g de carvão ativado CarboActiv V-plus. O mesmo procedimento de separação e quantificação descrito para as cinéticas de adsorção foi empregado.

\section{Influência do pH}

O efeito do pH no processo de adsorção foi testado em sistemas contendo 6,0 mL de solução com concentração $50 \mathrm{mg} \mathrm{L}^{-1} \mathrm{de} \mathrm{Pb}(\mathrm{II}) \mathrm{em}$ 0,1 g de carvão ativado Clarimex $061 \mathrm{CAE}$ ou $6,0 \mathrm{~mL}$ de solução de concentração $594 \mathrm{mg} \mathrm{L}^{-1}$ de $\mathrm{Pb}$ (II) em $0,04 \mathrm{~g}$ de carvão ativado CarboActiv V-plus, a $25^{\circ} \mathrm{C}$. $\mathrm{O} \mathrm{pH}$ foi ajustado para valores na faixa de 1,0 a 6,0 pela adição de soluções $0,1 \mathrm{~mol} \mathrm{~L}^{-1}$ de $\mathrm{NaOH}$ ou $\mathrm{HCl}$.

\section{Dessorção}

A dessorção foi avaliada em uma amostra de carvão CarboActiv V-plus previamente submetida ao processo de adsorção descrito com uma solução de concentração $594 \mathrm{mg} \mathrm{L}^{-1}$ de $\mathrm{Pb}$ (II). Uma amostra contendo $0,8 \mathrm{~g}$ deste carvão, seco em estufa, foi posta em contato com 120,0 mL de uma solução de $\mathrm{HCl} 1 \mathrm{~mol} \mathrm{~L}^{-1}$. O sistema foi mantido sob agitação magnética e foram recolhidas alíquotas de 5,0 $\mathrm{mL}$ nos intervalos de tempo na faixa de 1 a $20 \mathrm{~min}$. A concentração $\mathrm{de} \mathrm{Pb}$ (II) foi avaliada conforme descrito. O procedimento foi repetido para o carvão ativo Clarimex 061 CAE, após adsorção de uma solução contendo $50 \mathrm{mg} \mathrm{L}^{-1} \mathrm{de} \mathrm{Pb}(\mathrm{II})$. 


\section{RESULTADOS E DISCUSSÃO}

\section{Titulação de Boehm}

A titulação de Boehm tem seus resultados apresentados na Tabela 1.

É geralmente aceito que o hidrogenocarbonato de sódio neutraliza apenas grupos carboxílicos $(\mathrm{pK}<6,37)$, enquanto o carbonato de sódio titula grupos carboxílicos e lactonas ( $\mathrm{pK}<10,25)$. Hidróxido de sódio neutraliza grupos carboxílicos, lactonas e grupos fenólicos ( $\mathrm{pK}<15,74)$. Etóxido de sódio $(\mathrm{pK}=20,58)$ deve promover a neutralização total, incluindo grupos carbonílicos e não carbonílicos ${ }^{17}$. A titulação de Boehm mostra que os adsorventes possuem funcionalidades ácidas na superfície, através de grupos nãocarbonílicos (carboxílicos, fenólicos e lactonas) e grupos carbonílicos. Pode-se observar que os grupos não-carbonílicos estão presentes em quantidades semelhantes nos dois carvões. Entretanto, no carvão Clarimex estes grupos estão representados principalmente por lactonas e grupos fenólicos, grupos funcionais relativamente fracos que se dissociam em valores de $\mathrm{pH}$ mais altos que os grupos carboxílicos.

A quantidade total de grupos funcionais presentes nas amostras é semelhante, mas o carvão CarboActiv V-plus possui grupos carboxílicos em quantidade muito superior. Esse fato passa a ser importante, se considerarmos que os cátions metálicos podem estar sendo retidos por troca iônica na superfície do carvão. Os grupos carboxílicos, quando presentes em maior quantidade, facilitam o processo de adsorção, principalmente se o $\mathrm{pH}$ de trabalho estiver situado na faixa que favorece a desprotonação destes grupos.

\section{Titulação de pH}

As curvas de afinidade de prótons estão apresentadas na Figura 1 para os carvões Clarimex 061 e CarboActiv V-plus. Valores positivos nessas isotermas indicam a retenção de prótons. Valores negativos significam a liberação de prótons ou a ligação de íons hidroxila.

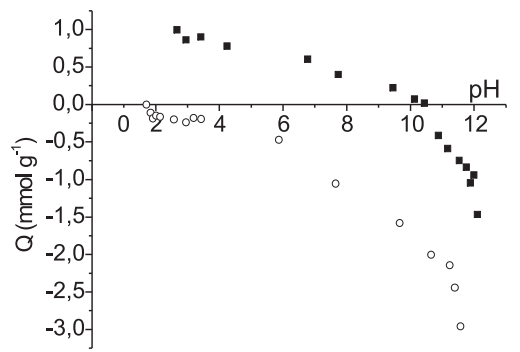

Figura 1. Isoterma de afinidade de prótons para o carvão Clarimex 061 ( ( ) e CarboActiv V-plus (

A curva para o carvão Clarimex 061 indica que essa amostra possui uma superfície heterogênea com total contribuição de grupos ácidos, enquanto para o carvão CarboActiv V-plus tem-se um comportamento anfótero, com contribuição tanto de grupos ácidos quanto básicos na superfície. O comportamento observado para o carvão CarboActiv V-plus é típico de carvões do tipo H, obtidos por exposi- ção ao vapor ou $\mathrm{CO}_{2}$ em elevadas temperaturas. Estes carvões, quando imersos em água, adsorvem íons $\mathrm{H}^{+}$em uma ampla faixa de $\mathrm{pH}$, tornando a sua superfície carregada positivamente ${ }^{18}$. De acordo com os resultados obtidos, este comportamento é predominante em valores de $\mathrm{pH}$ de até 10,5. Por outro lado, o processo de ativação do carvão Clarimex 061 é tipicamente para a produção de sólidos do tipo L, no qual se tem uma superfície carregada negativamente em solução aquosa, devido a adsorção de hidroxilas ${ }^{19}$. Neste caso, é esperada uma predominância de sítios ácidos, o que está de acordo com os resultados obtidos na titulação de $\mathrm{pH}$ da amostra.

As curvas de afinidade de prótons são relativamente suaves e não apresentam pontos de inflexão acentuados, característica de trocadores polifuncionais. Contudo, pode-se observar que o gradiente aumenta à medida que o $\mathrm{pH}$ também aumenta. Esse comportamento é notável principalmente em valores de $\mathrm{pH}$ superiores a 6,0 para o carvão Clarimex. À medida que o pH aumenta, grupos funcionais fracos como lactonas e fenóis começam a dissociar contribuindo para a capacidade de troca iônica do material adsorvente ${ }^{16}$. Já o carvão CarboActiv apresenta uma considerável quantidade de grupos passíveis de participação em processos de adsorção, mesmo em valores de $\mathrm{pH}$ reduzidos. Este fato é de grande relevância, uma vez que as soluções de $\mathrm{Pb}$ (II) apresentam caráter ácido, que aumenta com a sua concentração (na faixa de concentração estudada neste trabalho, os valores originais de $\mathrm{pH}$ variaram de 5,8 a 5,0).

A presença de grupos funcionais de superfície, como os anteriormente citados, tem sido relacionada com a fonte de acidez superficial. Por outro lado, a origem de propriedades básicas presentes na superfície de carvões é mais controversa e costuma ser relacionada à própria estrutura e distribuição dos átomos de carbono no sólido ${ }^{20}$ e à presença de pironas e íons superóxido ${ }^{21}$. A quantidade de grupos básicos foi estimada por neutralização com $\mathrm{HCl} 0,1 \mathrm{~mol} \mathrm{~L}^{-1}$. A amostra de carvão CarboActiv V-plus apresentou 0,997 meq de grupos básicos por grama, enquanto o carvão Clarimex não apresentou qualquer basicidade detectável, confirmando que a sua superfície tem propriedades essencialmente ácidas. $\mathrm{O}$ ponto de crossover ou ponto de carga zero $\left(\mathrm{pH}_{\mathrm{PZC}}\right)$ é uma medida da acidez ou basicidade total da superfície do adsorvente. Amostras oxidadas tendem a apresentar o seu ponto de crossover em valores reduzidos de $\mathrm{pH}$, como demonstrado pelo carvão Clarimex $\left(\mathrm{pH}_{\mathrm{PZC}}=1,7\right) \mathrm{e}$, quando o grau de oxidação diminui, o ponto de crossover ocorre em valores de $\mathrm{pH}$ superiores (carvão CarboActiv V-plus, $\left.\mathrm{pH}_{\mathrm{PZC}}=10,5\right)$.

A forma de ativação afeta profundamente as propriedades de sorção de cátions metálicos pelo carvão. Inicialmente, uma elevada capacidade de sorção pode ser atribuída ao caráter ácido dos grupos funcionais de superfície, que tendem a desprotonar na região de $\mathrm{pH}$ ácido a neutro e, portanto, favorecem a ligação de cátions metálicos. Entretanto, vários outros modelos de adsorção podem ser utilizados para descrever as propriedades de sorção destes materiais, dentre os quais troca iônica e complexação.

\section{Cinéticas de adsorção}

Os resultados de retenção de $\mathrm{Pb}(\mathrm{II})$ obtidos durante o acompanhamento cinético do processo de adsorção pelos carvões Clarimex 061 CAE e CarboActiv V-plus estão apresentados na Figura 2. No

Tabela 1. Concentração de grupos funcionais na superfície (meq g $\mathrm{g}^{-1}$ )

\begin{tabular}{lcccccc}
\hline Carvão & $\begin{array}{c}\text { Grupos } \\
\text { Carboxílicos }\end{array}$ & Lactonas & $\begin{array}{c}\text { Grupos } \\
\text { Fenólicos }\end{array}$ & $\begin{array}{c}\text { Total } \\
\text { (não-carbonílicos) }\end{array}$ & $\begin{array}{c}\text { Grupos } \\
\text { carbonílicos }\end{array}$ & $\begin{array}{c}\text { Quantidade } \\
\text { Total }\end{array}$ \\
\hline Clarimex 061 & 0,110 & 0,235 & 0,125 & 0,470 & 0,010 & 0,480 \\
CarboActiv V-plus & 0,360 & 0,020 & 0,105 & 0,485 & 0,075 & 0,560 \\
\hline
\end{tabular}


caso do carvão CarboActiv V-plus, utilizando-se uma solução de $\mathrm{Pb}$ (II) com concentração inicial de $50 \mathrm{mg} \mathrm{L}^{-1}$ houve retenção total do metal já na primeira amostra analisada, impedindo o acompanhamento cinético do processo, razão pela qual o procedimento foi refeito com uma solução de concentração inicial $594 \mathrm{mg} \mathrm{L}^{-1}$.

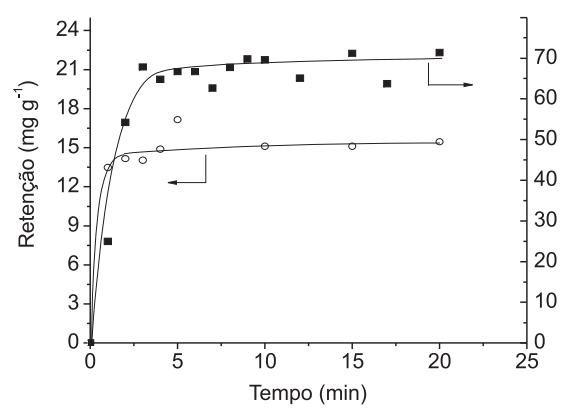

Figura 2. Retenção de Pb(II) pelo carvão Clarimex 061 (0) e CarboActiv V-plus (ロ) em função do tempo

Pode-se observar que a velocidade de sorção é elevada no início do processo, o que provavelmente está relacionado ao elevado número de sítios de adsorção disponíveis na superfície dos carvões. Os resultados indicam que o equilíbrio é alcançado nos primeiros $5 \mathrm{~min}$ de contato entre o sólido e a solução de $\mathrm{Pb}$ (II), para ambos os carvões. Diferentemente do que é observado em outros sólidos adsorventes como as zeólitas, onde o equilíbrio é alcançado em períodos tão elevados quanto $24 \mathrm{~h}^{22}$, tem-se aqui uma indicação de que os sítios de adsorção no carvão estão bastante acessíveis, provavelmente situados em poros que não conferem impedimento estérico à aproximação das espécies metálicas. Assim, os cátions metálicos hidratados estão penetrando nos poros do carvão sem a necessidade de perda de moléculas de água de suas estruturas. Este comportamento está de acordo com estudos feitos por diversos autores, nos quais se observou que não há influência da energia de solvatação dos cátions no processo de adsorção em carvões ${ }^{23}$.

Os processos de ativação alteram substancialmente a superfície dos carvões. Esta modificação pode ser relacionada a diversos fatores, tais como o bloqueio na entrada dos poros por grupos funcionais oxigenados e por moléculas de compostos húmicos residuais. A erosão na superfície do carvão causada pelo ataque oxidante também é outro fator determinante. O material cujo volume microporoso é o mais relevante corresponde ao carvão CarboActiv V-plus, conforme pode ser observado na Tabela 2. Para esta amostra, o raio médio dos microporos está em torno de 6,0 A. Considerando que o raio hidratado do $\mathrm{Pb}(\mathrm{II})$ é de $4,01 \AA^{24}$, podemos confir-

Tabela 2. Resultados de área superficial $\left(\mathrm{m}^{2} \mathrm{~g}^{-1}\right)$ e de volume de poros $\left(\mathrm{cm}^{3} \mathrm{~g}^{-1}\right)$ para os carvões ativados

\begin{tabular}{lccccc}
\hline Carvão & Área & \multicolumn{2}{c}{ Microporos } & \multicolumn{2}{c}{ Mesoporos } \\
& BET & Área & Volume & Área & Volume \\
\hline Clarimex 061 & 902 & 290 & 0,13 & 485 & 0,63 \\
CarboActiv V-plus & 872 & 414 & 0,21 & 303 & 0,52 \\
\hline
\end{tabular}

mar que o impedimento estérico não é fator predominante na limitação do processo de adsorção dos cátions metálicos.

Dois modelos cinéticos foram utilizados para avaliar os dados experimentais. Os modelos de Lagergren de pseudo-primeira ordem e de pseudo-segunda ordem foram testados de acordo com as equações apresentadas por Ho e $\mathrm{McKay}^{25}$.

A equação de pseudo-primeira ordem está representada na Equação 2.

$\log \left(q_{e}-q_{t}\right)=\log q_{e}-\frac{k_{1}}{2,203} t$

onde $\mathrm{k}_{1}$ é a constante de pseudo-primeira ordem, $\mathrm{q}_{\mathrm{e}}$ é a quantidade adsorvida no equilíbrio e $\mathrm{q}_{\mathrm{t}}$ a quantidade adsorvida em qualquer tempo $\mathrm{t}$.

O modelo de pseudo-segunda ordem é representado pela Equação 3

$\frac{t}{q_{t}}=\frac{1}{k_{2} q_{e}^{2}}+\frac{1}{q_{e}} t$

onde $\mathrm{k}_{2}$ é a constante de velocidade de pseudo-segunda ordem. $\mathrm{O}$ produto $\mathrm{k}_{2} \mathrm{q}_{\mathrm{e}}^{2}$ corresponde à velocidade inicial de adsorção, representada por $h_{0}$.

A validade desses modelos foi verificada através dos gráficos lineares $\log \left(\mathrm{q}_{\mathrm{e}}-\mathrm{q}_{\mathrm{t}}\right)$ versus $\mathrm{t}$ para o modelo da pseudo-primeira ordem e $\mathrm{t} / \mathrm{q}_{\mathrm{t}}$ versus $\mathrm{t}$ para o modelo de pseudo-segunda ordem. Os coeficientes de correlação obtidos (Tabela 3) indicaram que as cinéticas de adsorção seguem o modelo de pseudo-segunda ordem, onde os valores de $q_{e}$ e $k_{2}$ podem ser obtidos, respectivamente, através dos coeficientes angular e linear da linearização de $t / \mathrm{q}_{\mathrm{t}}$ versus $t$.

Observa-se que os valores de $\mathrm{q}_{\mathrm{e}}$ obtidos pela equação de pseudosegunda ordem estão de acordo com os valores de $\mathrm{q}_{\mathrm{e}}$ obtidos experimentalmente, o que também confirma a validade desse modelo em relação aos carvões avaliados. Portanto, os resultados obtidos aplicando-se o modelo cinético de Lagergren de pseudo-segunda ordem foram utilizados nos cálculos termodinâmicos apresentados a seguir.

\section{Isotermas de adsorção}

Os resultados experimentais obtidos na adsorção de $\mathrm{Pb}(\mathrm{II})$ pelos carvões Clarimex 061 e CarboActiv V-plus, a partir de soluções com diferentes concentrações do metal estão apresentados na Figura 3.

Os dados de equilíbrio foram processados de acordo com as isotermas de Langmuir e Freundlich ${ }^{26}$. Os parâmetros obtidos aplicando-se estes modelos aos dados experimentais são apresentados na Tabela 4.

Os resultados demonstram que o equilíbrio é mais bem descrito pelas isotermas de Langmuir, cujos coeficientes de correlação são elevados. As curvas estão caracterizadas por uma inclinação que diminui com o aumento da concentração de $\mathrm{Pb}$ (II) em solução, uma vez que a quantidade de sítios de adsorção livres diminui à medida em que a superfície do adsorvente é recoberta pelos cátions metálicos. Este comportamento pode justificar a elevada afinidade do adsorvente pelos cátions de $\mathrm{Pb}(\mathrm{II})$ em baixas concentrações. Os valores de $\mathrm{Q}_{0}$, constante relacionada à capacidade de adsorção

Tabela 3. Parâmetros cinéticos e coeficientes de correlação para a adsorção sobre os carvões ativados

\begin{tabular}{lccccc}
\hline Carvão & $\mathrm{q}_{\mathrm{e}, \text { exp }}$ & \multicolumn{2}{c}{ Pseudo-primeira ordem } & \multicolumn{3}{c}{ Pseudo-segunda ordem } \\
& $\left(\mathrm{mg} \mathrm{g}^{-1}\right)$ & $\mathrm{k}_{1}\left(\mathrm{~min}^{-1}\right)$ & $\mathrm{r}$ & $\mathrm{k}_{2}\left(\mathrm{~g} \mathrm{mg}^{-1} \mathrm{~min}^{-1}\right)$ & $\mathrm{q}_{\mathrm{e}}\left(\mathrm{mg} \mathrm{g} \mathrm{g}^{-1}\right)$ \\
\hline Clarimex 061 & 15,46 & 0,0092 & 0,1865 & 0,6048 & 15,48 \\
CarboActiv V-plus & 71,37 & 0,0701 & 0,3375 & 0,0210 & 71,94 \\
\hline
\end{tabular}


Tabela 4. Parâmetros característicos e coeficientes calculados a partir das equações de Langmuir e Freundlich para a retenção de $\mathrm{Pb}(\mathrm{II})$ a $25{ }^{\circ} \mathrm{C}$

\begin{tabular}{|c|c|c|c|c|c|c|c|}
\hline \multirow[t]{2}{*}{ Adsorvente } & \multicolumn{3}{|c|}{ Isoterma de Langmuir } & \multicolumn{3}{|c|}{ Isoterma de Freundlich } & \multirow[t]{2}{*}{ Ref. } \\
\hline & $\mathrm{Q}_{0}\left(\mathrm{mg} \mathrm{g}^{-1}\right)$ & $\mathrm{b}\left(\mathrm{L} \mathrm{mg}^{-1}\right)$ & $\mathrm{r}$ & $1 / \mathrm{n}$ & $\mathrm{K}_{\mathrm{f}}\left(\mathrm{mg}^{1-1 / \mathrm{n}} \mathrm{L}^{1 / \mathrm{n}} \mathrm{g}^{-1}\right)$ & $\mathrm{r}$ & \\
\hline Clarimex 061 & 11,5 & 0,02032 & 0,9990 & 0,57 & 0,44 & 0,9717 & Este trabalho \\
\hline CarboActiv V-plus & 69,4 & 0,02957 & 0,9933 & 0,37 & 6,72 & 0,9660 & Este trabalho \\
\hline $\mathrm{CA}$ & 25,5 & 0,8 & 0,9456 & 0,24 & 11,4 & 0,9959 & 27 \\
\hline $\mathrm{CA}$ & 45,1 & 1,695 & 0,9980 & 0,38 & 7,47 & 0,9757 & 28 \\
\hline $\mathrm{CA}$ & 30,1 & 0,0651 & 0,9698 & 3,85 & 6,46 & 0,9853 & 29 \\
\hline CA & 26,5 & 1,00 & 0,9996 & 0,56 & 6,01 & 0,9692 & 30 \\
\hline Palha & 11,9 & 0,0723 & 0,989 & 0,55 & 1,105 & 0,954 & 31 \\
\hline Cinzas & 12,6 & 0,1630 & 0,9979 & 0,31 & 3,499 & 0,9761 & 32 \\
\hline
\end{tabular}

$\mathrm{CA}=$ carvão ativado; $\mathrm{Q}_{0}=$ constante relacionada à capacidade de adsorção máxima, $\mathrm{b}=$ constante relacionada à energia de adsorção, $\mathrm{Kf}=$ medida da capacidade de adsorção, $1 / \mathrm{n}=$ medida da intensidade de adsorção, $\mathrm{r}=$ coeficiente de correlação.

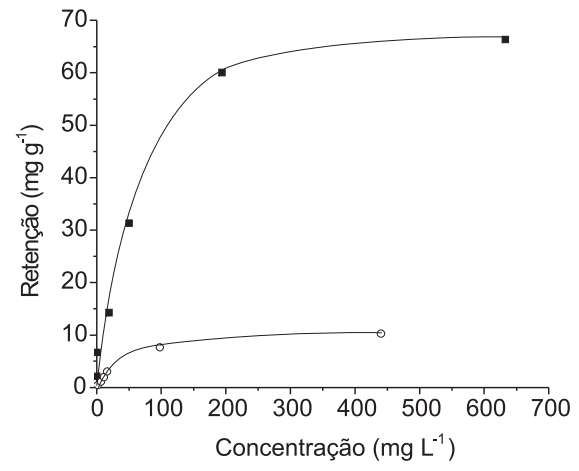

Figura 3. Retenção de Pb(II) pelo carvão Clarimex 061 (O) e CarboActiv Vplus (ם) em função da concentração de equilíbrio

máxima, estão condizentes com os resultados práticos obtidos. Devemos considerar que a maioria das suposições originalmente relacionadas ao modelo de Langmuir (adsorção em superfícies homogêneas com um número fixo de sítios de adsorção idênticos, reversibilidade, mesma energia de adsorção para todos os sítios) é inválida para a superfície heterogênea encontrada em carvões ativados. Assim, a equação de Langmuir deve ser usada preferencialmente para fins qualitativos e descritivos, enquanto uma análise quantitativa deve ser feita com precaução. Mesmo considerando estas limitações, muitos autores têm demonstrado que este modelo descreve adequadamente comportamentos de equilíbrio em diversos materiais, dentre os quais carvões ativados ${ }^{33}$. Embora os parâmetros de adsorção sejam dependentes de vários fatores, tais como área superficial e tamanho das partículas do adsorvente, $\mathrm{pH}$ da solução e velocidade de agitação, dentre outras condições operacionais, uma comparação entre os dados obtidos neste trabalho e valores encontrados na literatura também é feita na Tabela 4. Pode-se observar que o carvão CarboActiv V-plus demonstra a maior capacidade de adsorção dentre os trabalhos citados, o que está relacionado à elevada quantidade de grupos funcionais de superfície (principalmente grupos carboxílicos) presentes neste carvão. Em todos os trabalhos citados na Tabela 4, os autores identificaram como adequado o modelo de Langmuir na descrição do comportamento observado.

\section{Influência da temperatura}

Os estudos de adsorção foram feitos nas temperaturas de 5, 15, 25 e $50{ }^{\circ} \mathrm{C}$, para soluções com concentração inicial de $50 \mathrm{mg} \mathrm{L}^{-1}$ (Clarimex 061) e $594 \mathrm{mg} \mathrm{L}^{-1}$ (CaboActiv V-plus), mantendo-se os valores de $\mathrm{pH}$ originais. Os resultados da retenção de $\mathrm{Pb}$ (II) em fun- ção da temperatura foram tratados de acordo com o modelo cinético de pseudo-segunda ordem de Lagergren e apresentados na Tabela 5.

Tabela 5. Influência da temperatura no processo de adsorção, de acordo com o modelo de pseudo-segunda ordem

\begin{tabular}{lccccc}
\hline Carvão & $\begin{array}{c}\text { Temperatura } \\
\left({ }^{\circ} \mathrm{C}\right)\end{array}$ & $\begin{array}{c}\mathrm{q}_{\mathrm{e}} \\
\left(\mathrm{mg} \mathrm{g}^{-1}\right)\end{array}$ & $\begin{array}{c}\mathrm{k}_{2} \\
\left(\mathrm{~g} \mathrm{mg}^{-1}\right. \\
\left.\mathrm{min}^{-1}\right)\end{array}$ & $\begin{array}{c}\mathrm{h}_{0} \\
\left(\mathrm{mg} \mathrm{g}^{-1}\right.\end{array}$ & $\mathrm{r}$ \\
$\left.\mathrm{min}^{-1}\right)$ & \\
\hline Clarimex 061 & 5 & 1,54 & 0,6059 & 1,44 & 0,9999 \\
& 15 & 1,59 & 0,7163 & 1,83 & 0,9991 \\
& 25 & 1,61 & 0,8510 & 2,19 & 0,9917 \\
CarboActiv & 50 & 1,79 & 1,9874 & 6,39 & 0,9998 \\
V-plus & 5 & 43,48 & 0,0235 & 44,44 & 0,9932 \\
& 15 & 51,28 & 0,0400 & 105,26 & 0,9973 \\
& 25 & 68,97 & 0,0309 & 147,06 & 0,9886 \\
& 50 & 70,92 & 0,0947 & 476,19 & 0,9969 \\
\hline
\end{tabular}

É possível identificar um significativo aumento na capacidade de adsorção com o aumento da temperatura, o que pode ser confirmado pelos valores de $\mathrm{q}_{\mathrm{e}}$ obtidos a partir do modelo cinético de segunda ordem. Do mesmo modo, o aumento na velocidade inicial de adsorção é elevado, principalmente para o carvão CarboActiv. Estes resultados demonstram que a adsorção do $\mathrm{Pb}$ (II) pelos carvões avaliados neste trabalho é um processo endotérmico, o que está de acordo com os resultados obtidos por Corapcioglu e Huang ${ }^{17}$ na adsorção de $\mathrm{Cu}(\mathrm{II})$ sobre carvão ativado, Ho e colaboradores ${ }^{34}$ na adsorção de $\mathrm{Pb}$ (II) em vegetais, Chantawong e colaboradores ${ }^{35}$ na adsorção de $\mathrm{Cd}(\mathrm{II})$ em "ballclay" e em Panayotova ${ }^{36}$ com $\mathrm{Cu}$ (II) em zeólitas. A energia de ativação aparente calculada pela maneira usual, usando a equação de Arrhenius, é de 4,80 e 5,23 kcal mol${ }^{-1}$, respectivamente, para os carvões Clarimex e CarboActiv.

Os resultados de adsorção obtidos com o carvão Clarimex 061 podem ser relacionados ao tipo e à quantidade de grupos funcionais de superfície presentes no carvão, assim como determinado pelo método de Bohem. Este carvão apresenta uma superfície que resulta em uma baixa capacidade de retenção de cátions, sendo que o valor de $\mathrm{Q}_{0}$ é bastante inferior à concentração total de grupos de superfície. Devemos considerar que os valores de $\mathrm{pH}$ das soluções de $\mathrm{Pb}$ (II) são inferiores a 5,8 e, portanto, uma parcela pouco significativa do total de grupos ácidos de superfície está disponível para ligação dos cátions metálicos. Isto pode ser confirmado nas isotermas de afinidade de prótons (Figura 1), onde se observa uma reduzida quantidade de grupos ácidos desprotonados nesta faixa de $\mathrm{pH}$.

A maior capacidade de retenção é identificada para o carvão CarboActiv V-plus, cuja caracterização indicou ser uma amostra 
com reduzida quantidade de grupos carbonílicos, mas com quantidade superior de grupos carboxílicos.

De acordo com Weber e Digiano ${ }^{37}$, os principais modelos de adsorção podem ser representados pelos fenômenos de atração elétrica e de atração química. A adsorção por atração elétrica envolve principalmente os processos de troca iônica, enquanto na atração química temos o adsorvato ligando-se quimicamente ao adsorvente. Diante dos resultados obtidos, podemos supor que o mecanismo de troca iônica é predominante, principalmente no caso do carvão CarboActiv V-plus. Os processos de troca iônica envolvem energias de adsorção superiores àquelas identificadas nas atrações por forças de van der Waals $\left(1,2\right.$ a 2,3 $\left.\mathrm{Kcal} \mathrm{mol}^{-1}\right)$, enquanto na atração química estão envolvidas elevadas energias de adsorção, tipicamente de 25 a $100 \mathrm{Kcal} \mathrm{mol}^{-138}$. Além disso, o fenômeno de troca iônica é considerado reversível ${ }^{39}$.

Devemos ainda considerar que o processo de ativação do carvão aumenta a concentração dos grupos de superfície não carboxílicos. Estes grupos podem promover a formação de complexos de esfera externa, nos quais a interação se dá através de ligações de hidrogênio, menos energéticas que as ligações covalentes. Conseqüentemente, temos interações cujo valor da energia de ativação não é tão elevado quanto aqueles observados em atrações químicas típicas. Portanto, a interação de cátions metálicos com a superfície do sólido por atração química, do tipo ligação de hidrogênio, pode não ser a principal responsável pela retenção de $\mathrm{Pb}(\mathrm{II})$, mas não deve ser desconsiderada.

Os resultados obtidos com as isotermas de adsorção foram utilizados no cálculo de parâmetros termodinâmicos. O calor de adsorção $\Delta \mathrm{H}^{0}$, e a variação de entropia, $\Delta \mathrm{S}^{0}$, foram avaliados de acordo com a expressão:

$\ln K_{d}=\frac{\Delta S^{0}}{R}-\frac{\Delta H^{0}}{R T}$

onde $\mathrm{R}$ é a constante geral dos gases $\left(8,31451 \mathrm{~J} \mathrm{~K}^{-1} \mathrm{~mol}^{-1}\right)$, $\mathrm{T}$ é a temperatura (em Kelvin) e $K_{d}$ é o coeficiente de distribuição, definido como a concentração de $\mathrm{Pb}$ (II) adsorvido por grama de adsorvente dividido pela sua concentração na fase líquida. Os valores de $\Delta \mathrm{H}^{0}$ e $\Delta \mathrm{S}^{0}$ foram calculados, respectivamente, a partir dos coeficientes angular e linear da reta obtida no gráfico de $\ln \mathrm{K}_{\mathrm{d}}$ versus 1/T. A energia livre de Gibbs $\Delta \mathrm{G}^{0}$, foi determinada de acordo com:

$\Delta G^{0}=\Delta H^{0}-T \Delta S^{0}$

A Tabela 6 reúne os valores termodinâmicos calculados.
Os valores encontrados para $\Delta \mathrm{G}^{0}, \Delta \mathrm{H}^{0}$ e $\Delta \mathrm{S}^{0}$ indicam a espontaneidade do processo de adsorção. Os valores de $\Delta \mathrm{H}^{0}$ são positivos, confirmando a natureza endotérmica do processo de sorção, como já havia sido demonstrado pelos valores das constantes de equilíbrio. Porém, os valores de $\Delta \mathrm{H}^{0}$ são reduzidos, o que reforça a hipótese de que a interação química, do tipo ligação de hidrogênio, tem um papel secundário, mas significativo na retenção de $\mathrm{Pb}(\mathrm{II}) . \Delta \mathrm{S}^{0}$ é positivo e sugere que a desordem na interface sólido-solução aumenta após a adsorção do $\mathrm{Pb}(\mathrm{II})$. Este comportamento pode ser relacionado às substituições que ocorrem na esfera de coordenação dos íons metálicos, de modo que seja possível a ocorrência das interações químicas necessárias ao processo de adsorção na superfície do carvão. Íons $\mathrm{Pb}(\mathrm{II})$ formam complexos lábeis em sistemas aquosos $^{43}$, o que contribui para reduzir o tempo necessário para alcançar o equilíbrio de adsorção. Quando comparados aos valores encontrados na literatura, os dados obtidos neste trabalho são semelhantes (tanto a ordem de grandeza quanto o sinal). A diferença fica por conta dos dados obtidos por Pehlivan e Arslan ${ }^{41}$ e por Han et l. $^{31}$, que identificaram um processo exotérmico. Os autores justificaram este comportamento em razão da complexação ser o principal mecanismo envolvido na retenção dos cátions pela lignita e pela palha utilizadas como adsorventes, o que não corresponde ao comportamento observado para o carvão CarboActiv V-plus testado neste trabalho.

\section{Influência do pH}

Os resultados dos testes de influência do $\mathrm{pH}$ na retenção de $\mathrm{Pb}$ (II) são apresentados na Figura 4.

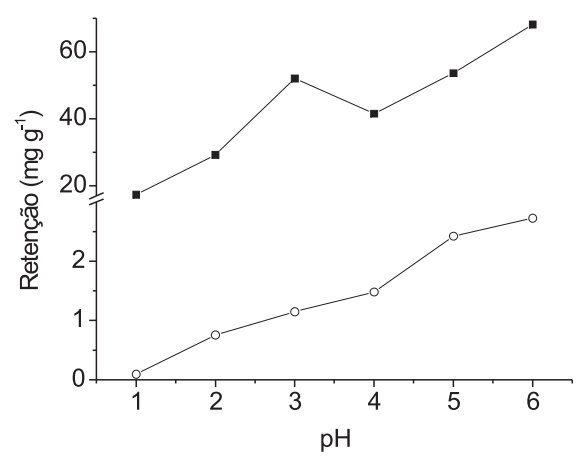

Figura 4. Influência do pH na adsorção de Pb(II) pelos carvões ativados Clarimex 061 (O) e CarboActiv V-plus (ロ)

Tabela 6. Parâmetros termodinâmicos obtidos a partir das isotermas de adsorção

\begin{tabular}{|c|c|c|c|c|c|c|}
\hline Adsorvente & Temperatura $\left({ }^{\circ} \mathrm{C}\right)$ & Retenção(\%) & $\Delta \mathrm{G}^{0}\left(\mathrm{~kJ} \mathrm{~mol}^{-1}\right)$ & $\Delta \mathrm{H}^{0}\left(\mathrm{~kJ} \mathrm{~mol}^{-1}\right)$ & $\Delta \mathrm{S}^{0}\left(\mathrm{~J} \mathrm{~K}^{-1} \mathrm{~mol}^{-1}\right)$ & Ref. \\
\hline \multirow[t]{4}{*}{ Clarimex 061} & 5 & 56,5 & $-10,00$ & 4,46 & 52,0 & Este trabalho \\
\hline & 15 & 59,3 & $-10,52$ & & & \\
\hline & 25 & 60,2 & $-11,04$ & & & \\
\hline & 50 & 51,0 & $-12,34$ & & & \\
\hline \multirow[t]{4}{*}{ CarboActiv V-plus } & 5 & 48,8 & $-12,52$ & 16,98 & 106,1 & Este trabalho \\
\hline & 15 & 62,0 & $-13,58$ & & & \\
\hline & 25 & 74,9 & $-14,64$ & & & \\
\hline & 50 & 77,5 & $-17,29$ & & & \\
\hline $\mathrm{Fe} / \mathrm{Al}_{2} \mathrm{O}_{3}$ & 15 & - & $-6,85$ & 25,73 & 113,0 & 40 \\
\hline Lignita & 25 & - & $-4,83$ & $-0,097$ & $-316,0$ & 41 \\
\hline CA & 25 & - & $-6,07$ & 1,84 & 26,6 & 42 \\
\hline $\mathrm{CA}$ & 35 & - & $-2,796$ & 93,42 & 312,3 & 30 \\
\hline Palha & 25 & - & $-4,46$ & $-13,6$ & $-31,0$ & 31 \\
\hline
\end{tabular}

$\overline{\mathrm{CA}}=$ carvão ativado 
O comportamento dos dois carvões foi semelhante. A capacidade de troca iônica é fortemente influenciada pelos valores de $\mathrm{pH}$ da solução, pois estes determinam a especiação do metal a ser adsorvido. Com o aumento do $\mathrm{pH}$, poderíamos supor a presença de maior concentração de complexos nos quais moléculas de água são substituídas por outros ligantes inorgânicos, como $\mathrm{OH}^{-}$. Estas estruturas, por serem maiores ou menos solúveis, dificultariam o processo de troca iônica ${ }^{44}$. Entretanto, uma avaliação da especiação do metal indica que tais complexos ou mesmo compostos insolúveis não são formados em quantidades significativas na faixa de $\mathrm{pH}$ na qual foram realizados os testes ${ }^{45}$.

A alteração predominante está relacionada com a concentração de íons $\mathrm{H}^{+}$em solução. Em valores reduzidos de $\mathrm{pH}$, temos elevadas concentrações de $\mathrm{H}^{+}$, o que aumenta a competição pelos sítios de troca no carvão e dificulta a retenção dos cátions metálicos. Porém, como o aumento do $\mathrm{pH}$ se caracteriza pela diminuição na concentração de íons $\mathrm{H}^{+}$na solução (diminuindo, dessa forma, a competição destes com os cátions do metal), o processo é otimizado em valores de $\mathrm{pH}$ para os quais ocorre menor protonação dos sítios de troca do carvão.

\section{Dessorção}

Os resultados de dessorção são apresentados na Figura 5.

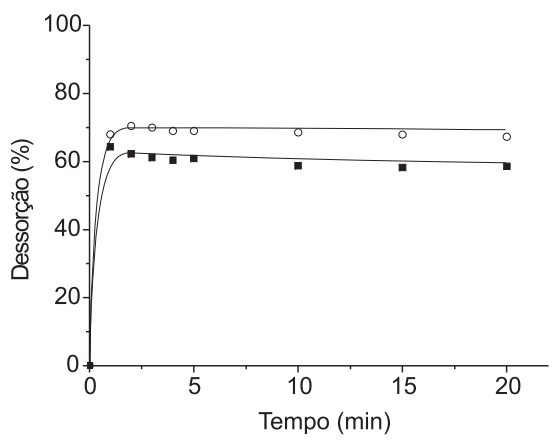

Figura 5. Dessorção de $\mathrm{Pb}(\mathrm{II})$ mediante tratamento dos carvões Clarimex 061 ( ( ) e CarboActiv V-plus ( $\mathbf{\square}$ ) previamente saturados com $\mathrm{Pb}(\mathrm{II})$, com $\mathrm{HCl}$ $1 \mathrm{~mol} \mathrm{~L}^{-1}$

Os carvões demonstraram uma capacidade de dessorção entre 65 e $70 \%$ do metal adsorvido. O tempo de equilíbrio necessário para a dessorção é bastante reduzido, tipicamente inferior a 5 min. Embora as porcentagens de dessorção sejam semelhantes, quando avaliamos as quantidades adsorvidas e dessorvidas, podemos observar que o carvão CarboActiv V-plus demonstra maior capacidade de regeneração. Foi possível recuperar cerca de $41 \mathrm{mg}$ de $\mathrm{Pb}$ (II) do total de $76 \mathrm{mg}$ retidos por grama de carvão. Enquanto isso, a recuperação no caso do carvão Clarimex 061 foi de apenas $1,8 \mathrm{mg}$ de $\mathrm{Pb}(\mathrm{II})$. Considerando que os processos de troca iônica são reversíveis, a dessorção deveria ter alcançado porcentagens mais elevadas como, por exemplo, a dessorção total observada com o uso de argilas ${ }^{10}$. Por outro lado, os cátions metálicos coordenados em sítios não carboxílicos podem estar contribuindo com a redução da porcentagem de dessorção, uma vez que estão mais fortemente presos à superfície dos sólidos.

\section{CONCLUSÕES}

Carvões têm importante aplicação em vários campos da proteção ambiental. $\mathrm{O}$ uso dos carvões testados para a remoção de $\mathrm{Pb}$ (II) presente em efluentes aquosos apresentou resultados promissores, porém o que mais se destacou nesta avaliação foi o CarboActiv Vplus. Este carvão apresenta uma superfície heterogênea, composta por sítios ácidos e básicos.

A temperatura influencia fortemente o processo de adsorção, de natureza endotérmica, o que foi demonstrado pelos valores das constantes de velocidade e das energias de ativação nos ensaios realizados.

Os testes de $\mathrm{pH}$ demonstraram que a retenção dos metais aumenta com o aumento do $\mathrm{pH}$, uma vez que nestas condições os sítios de troca estão menos protonados e, portanto, mais disponíveis para reter os cátions presentes em solução.

Nos testes de dessorção, tanto o carvão Clarimex 061 como o CarboActiv V-plus demonstraram capacidade de liberar parcialmente os cátions $\mathrm{Pb}(\mathrm{II})$ adsorvidos em suas estruturas, porém este último apresentou capacidade mais elevada quando comparado com o Clarimex 061.

Os resultados indicam que a natureza do processo de adsorção é predominantemente por troca iônica, sendo que a retenção devida à formação de complexos de esfera externa também é significativa.

Aliado aos fatos do carvão ocorrer em abundância e de não ser impactante ao meio ambiente, sua capacidade de adsorção torna o seu uso na retenção de íons metálicos, potencialmente tóxicos, de efluentes aquosos potencialmente útil economica e ecologicamente. Podemos prever uma utilização adequada do carvão CarboActiv V-plus em sistemas contínuos, nos quais o material é testado em colunas de adsorção, efetuando-se ciclos de adsorção/dessorção consecutivos.

\section{AGRADECIMENTOS}

Ao apoio financeiro concedido pelo CNPq (470458/04-6). C. I. de C. Bueno agradece à FAPESP pela bolsa concedida (05/04342-7).

\section{REFERÊNCIAS}

1. Salgado, P. E. T. Em Fundamentos de toxicologia; Oga, S., ed.; 2a . ed., Atheneu: São Paulo, 2003

2. Manahan, S. E.; Environmental Science and Technology, Lewis: New York, 1997.

3. Howard, A. G.; Aquatic Environmental Science and Technology, Lewis: New York, 1997.

4. Nerbitt, C. C.; Davis, T. E. Em Extraction and Processing for the Treatment and Minimization of Waste; Nerbitt, C. C., ed.; The Mineral, Metals and Materials Society: San Francisco, 1994.

5. Petroni, S. L. G.; Pires, M. A. F.; Munita, C. S.; Quim. Nova 2000, 23, 477; Luz Jr, G. E.; Guimarães Neto, J. M.; Moita Neto, J. M.; Quim. Nova 2005, 28, 535; Lamim, A. P. B.; Jordão, C. P.; Pereira, J. L.; Bellato, C. R.; Quim. Nova 2001, 24, 18 .

6. Santos, E. G.; Alsina, O. L. S.; Silva, F. L. H.; Quim. Nova 2007, 30, 327; Basílio, M. S.; Friese, K.; Lena, J. C.; Nalini Jr, H. A.; Roeser, H. M. P.; Quim. Nova 2005, 28, 822; Soares, J. P.; Souza, J. A.; Cavalheiro, E. T. G.; Quim. Nova 2004, 27, 5.

7. Cestari, A. R.; Vieira, E. F. S.; Matos, J. D. S.; Anjos, D. S. C.; J. Colloid Interface Sci. 2005, 285, 288; Lima, I. S.; Ribeiro, E. S.; Airoldi, C.; Quim. Nova 2006, 29, 501.

8. Jimenez, R. S.; Bosco, S. M. D.; Carvalho, W. A.; Quim. Nova 2004, $27,734$.

9. Barros, M. A. S. D.; Gazola, F. C.; Pereira, M. R.; Silva, E. A.; Arroyo, P. A.; Chem. Eng. J. 2006, 117, 253.

10. Bosco, S. M. D.; Jimenez, R. S.; Vignado, C.; Fontana, J.; Geraldo, B.; Figueiredo, F. C. A.; Mandelli, D.; Carvalho, W. A.; Adsorption 2006, 12, 133.

11. Gonçalves, A. R.; Benar, P.; Costa, S. M.; Ruzene, D. S.; Moriya, R. Y.; Luz, S. M.; Ferretti, L. P.; Appl. Biochem. Biotechnol. 2005, 121-24, 821.

12. Qin, F.; Wen, B.; Shan, X.-Q.; Xie, Y.-N.; Liu, T.; Zhang, S.-Z.; Khan, S. U.; Environ. Pollut. 2006, 144, 669; Falone, S. Z.; Vieira, E. M.; Quim. Nova 2004, 27, 849.

13. Lima, A. B.; Cardoso, M. G.; Guerreiro, M. C.; Pimentel, F. A.; Quim. Nova 2006, 29, 247.

14. Oda, H.; Nakagawa, Y.; Carbon 2003, 41, 1037.

15. Boehm, H. P.; Carbon 1994, 32, 759.

16. Strelko, V.; Malik, D. J.; J. Colloid Interface Sci. 2002, 250, 213.

17. Corapcioglu, M. O.; Huang, C. P.; Water Res. 1987, 21, 1031. 
18. Ishizaki, C.; Marti, I.; Carbon 1981, 19, 409.

19. Corapcioglu, M. O.; Huang, C. P.; Carbon 1987, 25, 569.

20. Barton, S. S.; Evans, M. J. B.; Halliop, E.; McDonald, J. A. F.; Carbon 1997, 35, 1361

21. Biniak, S.; Szymanski, G.; Siedlewski, J.; Swiatkowski, A.; Carbon 1997, 35, 1799.

22. Carvalho, W. A.; Jimenez, R. S.; Bosco, S. M. D.; J. Colloid Interface Sci. 2005, 281, 424

23. Youssef, A. M.; El-Nabarawy, T.; Samra, S. E.; Colloids Surf., A 2004, 235, 153.

24. Nightingale Jr., E. R.; J. Phys. Chem. 1959, 63, 1381.

25. Ho, Y. S.; McKay, G.; Trans. Inst. Chem. Eng. 1998, 76B, 332.

26. Hinz, C.; Geoderma 2001, 99, 225.

27. Rao, M. M.; Rao, G. P. C.; Seshaiah, K.; Choudary, N. V.; Wang, M. C.; Waste Manag. 2007, no prelo, disponível on line 9/4/2007, doi:10.1016/ j.wasman.2007.01.017.

28. Amud, O. S.; Giw, A. A.; Bello, I. A.; Biochem. Eng. J. 2007, 36, 174, doi:10.1016/j.bej.2007.02.013.

29. Mohan, D.; Pittman Jr., C. U.; Bricka, M.; Smith, F.; Yancey, B.; Mohammad, J.; Steele, P. H.; Franco, M. F. A.; Serrano, V. G.; Gong, H.; J. Colloid Interface Sci. 2007, 310, 57, doi:10.1016/j.jcis.2007.01.020.

30. Sekar, M.; Sakthi, V.; Rengaraj, S.; J. Colloid Interface Sci. 2004, 279, 307.

31. Han, R.; Zhang, J.; Zou, W.; Shi, J.; Liu, H.; J. Hazard Mater. B 2005, 125, 266.

32. Feng, Q.; Lin, Q.; Gong, F.; Sugita, S.; Shoya, M.; J. Colloid Interface Sci.
2004, 278, 1.

33. Álvarez-Ayuso, E.; Sánchez A. G.; Environ. Pollut. 2003, 125, 337; Matos, G. D.; Arruda, M. A. Z.; Process Biochem. 2003, 39, 81; Namasivayam, C.; Periasamy, K.; Water Res. 1993, 27, 1663.

34. Ho, Y.-S.; Chiu, W.-T.; Hsu, C.-S.; Huang, C.-T.; Hydrometallurgy 2004, $73,55$.

35. Chantawong, V.; Harvey, N. W.; Bashkin, V. N.; Water, Air, Soil Pollut. 2003 , $148,111$.

36. Panayotova, M. I.; Waste Manag. 2001, 21, 671.

37. Weber, W. J.; Digiano, F. A.; Process dynamics in environmental systems, Wiley: New York, 1996.

38. Jia, Y. F.; Steele, C. J.; Hayward, I. P.; Thomas, K. M.; Carbon 1998, 36, 1299

39. Bencheikh-Lehocine, M.; Environ. Technol. Lett. 1989, 10, 101.

40. Huang, Y.-H.; Hsue, C.-L.; Huang, C.-P.; Su, L.-C.; Chen, C.-Y.; Sep. Purif. Technol. 2007, 55, 23

41. Pehlivan, E.; Arslan, G.; Fuel Process. Technol. 2007, 88, 99.

42. Lia, Y.-H.; Dib, Z.; Ding, J.; Wu, D.; Luan, Z.; Zhu, Y.; Water Res. 2005, 39, 605.

43. van Leeuwen, H. P.; Town, R. M.; Buffle, J.; Cleven, R. F. M. J.; Davison, W.; Puy, J.; van Riemsdijk, W. H.; Sigg, L.; Environ. Sci. Technol. 2005, $39,8545$.

44. Ouki, S. K.; Kavannagh, M.; Water Sci. Technol. 1999, 39, 115.

45. Puigdomenech, I.; MEDUSA; Make Equilibrium Diagrams Using Sophisticated Algorithms; Royal Institute of Technology, Stockholm, Sweden, 2004. 